\title{
High Mode Vortex Induced Vibration (VIV) Experiments on a Large-Scale Riser
}

\author{
David Murrin*, Martin Ordonez*, Gillian Stone ${ }^{\dagger}$, Neil Bose ${ }^{\ddagger}$, and Wei Qiu* \\ ${ }^{*}$ Ocean and Naval Architectural Engineering \\ Memorial University of Newfoundland, St. John's, NL A1B 3X5 \\ ${ }^{\dagger}$ AMOG Consulting,Victoria, Australia, 3168 \\ $\ddagger$ Australian Maritime Hydrodynamics Research Centre (AMHRC), Newnham, Tasmania, 7248
}

\begin{abstract}
The focus of this research is on the development and testing of a large-scale model riser $(130 \mathrm{~m}$ in length) undergoing high mode vortex induced vibrations (VIV) in the ocean environment. This large scale model will provide an intermediate step between the common riser models $(8-10 \mathrm{~m}$ in length) that have mainly been used to research VIV to date and the actual $3,000+m$ deepwater risers being used in industry today.

During offshore drilling operations, marine risers carry mud and debris from below the sea floor, and during production operations they are responsible for transporting oil or gas from the hydrocarbon reservoir to the surface platform. The integrity of a marine riser is therefore critical to the success of offshore drilling and production.

The flow of seawater around marine risers is subject to vortex shedding which excites oscillations known as Vortex Induced Vibrations (VIV). When the VIV frequency approaches one of the natural frequencies of the structure, resonance, or lock-in occurs. This results in enhancement of the vibration amplitude of the structure and may have potentially destructive consequences due to high bending stresses and fatigue damage of the riser. At present, the prediction of this phenomenon is one of the most challenging areas in the offshore industry.

In experimental investigations of VIV, large aspect ratio risers, namely long cylinders with relatively small diameters, pose a modeling challenge. This paper focuses on the development of a large-scale model riser capable of transmitting data in real-time under realistic operating conditions.
\end{abstract}

\section{INTRODUCTION}

The phenomenon of vortex shedding around cylindrical structures is a concern in many different engineering fields. Vortex Induced Vibration (VIV) occurs when the vortex shedding frequency is approximately equal to the structures natural frequency. This phenomenon is of great importance to the offshore oil and gas industry due to its use of cylindrical risers to transport product and other fluids. There is a strong need to address deepwater issues on marine risers as offshore oil and gas exploration moves into ever increasing water depths High-mode VIV can occur with deepwater risers due to their excitation from current forces and high tension. While shallow water riser spans often experience only first mode vibration, deepwater risers can experience very high modes of vibration and can also experience more than one mode of vibration at a time. The focus of this research was to design, assemble, and test a large scale $(130 \mathrm{~m})$ deepwater riser model for VIV. This is valuable information for future analysis as a large scale model provides an intermediate step between the common $810 \mathrm{~m}$ riser models that have frequently been used to research VIV and the actual 3,000m deepwater risers being used in industry today. Also in this paper, a detailed description of the parts of the model as well as the assembly process is given along with the testing procedure. Finally, a summary of conclusions and recommendations have been included with regards to improving every aspect of the project, from designing to assembly and testing.

In 1976 [1] tested $23.3 \mathrm{~m}$ segments of various cables with fixed ends held under constant tension in a uniform flow. They found that as the Strouhal frequency approached the cables natural frequency, the phenomena of lock-in occurred. The maximum Reynolds number tested was 6850, and maximum mode number achieved was 7 . There were only 2 accelerometers used to measure model displacements. A second set of experiments were performed by [2] in 1983 at Reynolds up to 22000 , using the same apparatus, and results showed that the drag coefficient reached values larger than 3. Again, only the seventh mode was achieved, and only 7 pairs of accelerometers were used over the $23.3 \mathrm{~m}$ model length. Reference [3] hung a $267 \mathrm{~m}$ Kevlar cable over the side of a vessel and found that the vibration response of a long cable was essentially that of an infinite string and single mode lock-in was not observed in a shear current. Only 3 accelerometers were used to determine modal displacements and there were problems reading the inflowing current correctly. Reference [4] conducted sheared current tests on an instrumented cable arranged horizontally across the width of a $17.7 \mathrm{~m}$ canal. The test cable was a $0.028 \mathrm{~m}$ diameter rubber hose with 6 pairs of accelerometers along the length and a maximum mode number of 11 was achieved. Reference [5] conducted large scale tests involving two composite fiberglass pipes, $61 \mathrm{~m}$ and $122 \mathrm{~m}$ long, and $0.033 \mathrm{~m}$ in diameter. Twenty-four tri-axial accelerometers were spaced evenly along the length of the model and the maximum Reynolds number was approximately 34,000. The authors reported cross-flow flow excitation of up to the 25th mode.

Due to any number of causes, such as sparse instrumentation, small length to diameter ratios, low Reynolds numbers, low current velocities, high turbulence, and low maximum mode numbers of vibration, there are still many questions surrounding VIV of deepwater risers and there is still much 
work to be done in this area. The research presented in this paper is the design, development and implementation of a densely instrumented large scale riser model at a high Reynolds number and high maximum modes of vibration to produce valuable information about the behavior of long flexible deepwater risers responding to high mode vortex shedding.

\section{Methodology of Research}

\section{A. Design Considerations and Laboratory Testing}

An experimental apparatus of the size and scope necessary for large scale VIV testing requires considerable planning and testing. For this reason a small-scale test facility was built to evaluate design alternatives and develop installation procedures for the large scale riser. The design philosophy for the large-scale riser was based upon the principles of reliability, robustness, and Redundancy.

1) Reliability: The model riser is intended to serve as a test platform for a number of VIV experiments and it was important to ensure that the instrumentation produced strong and consistent signals. For this reason an industrial communication bus was used to ensure high-speed data transmission even under noisy electrical environments. The high bandwith and error checking protocol associated with this communication channel allowed for a larger number of sensor modules at high sampling rates to be used without sacrificing data integrity. A category 5 (cat5) network cable was chosen to facilitate communication between the instrumentation and data acquisition system. These cables are designed for high signal integrity, and include four twisted pairs in a single cable jacket. The test laboratory was used to evaluate the strength and consistency of a signal sent through the cat 5 cable over a length of $200 \mathrm{~m}$. This cable was further tested with a number of accelerometers along the $200 \mathrm{~m}$ length, and no degredation of the signal was evident.

2) Robustness: An important mechanical consideration is that of the forces experienced by the electronics due to the motion of the riser undergoing VIV. During field tests the riser is expected to experience large (and fluctuating) tension forces. It was important, therefore to design a system that would maintain the structural integrity of the system, and ensure that the delicate instruments and wire connections did not experience excessive loading. If a lateral tension force is applied to system of cables, the shortest cable in the bundle will bear the force applied to the system. In order to ensure that the cables and instrumentation inside the pipe did not feel a tension force applied to the riser it was necessary to direct the force along another cable designed to experience such loadings.

After a series of tests in the laboratory it was determined that the best arrangement consisted of a tensioned wire rope extending from one end of the riser to the other, onto which all of the electronic devices and accompanying wires were tightly mounted at fixed locations and somewhat slack elsewhere.
TABLE I

Riser PRIMARY DimENSIONS

\begin{tabular}{|c||c|}
\hline Model length $(\mathrm{m})$ & 130 \\
\hline Outer Diameter $(\mathrm{mm})$ & 53 \\
\hline Inner Diameter $(\mathrm{mm})$ & 40 \\
\hline Mass/length $(\mathrm{kg} / \mathrm{m})$ & 1.5 \\
\hline
\end{tabular}

3) Redundancy: In order to increase the reliability of an engineering system in the event of failure, critical components are duplicated to provide a backup or failsafe. This is known as Redundancy. It was important to build redundancy into the design of the model riser system, so that reliable data could be obtained in the event of one or more systems failing.

As previously discussed, the cat5 cable consists of four sets of twisted wire pairs. It was decided to design the instrumentation and data acquisition systems so that sensors could be staggered along the communication cable such that every fourth sensor module was connected to the same twisted pair. This provided for some redundancy in the event that one or more of the twisted pairs failed, since there would still be instruments spanning the entire length of the riser. The system was also designed using two power cables for similar reasons. The test laboratory was used to evaluate the system with a number of accelerometers staggered over each of the four twisted pairs of a $200 \mathrm{~m}$ cat5 network cable. The tests consisted of removing entire communication lines from the system, as well as removing individual sensor modules from each of the twisted pairs. The results showed a consistent and strong signal for each of the test cases.

Finally, after numerous design iterations, T-connections were eliminated from the electrical system and components were either soldered directly or tapped onto an existing conductor without interrupting service (using tap-connectors). The use of T-connectors required that the communications cable be cut at each sensor location, and joined to the sensor module. The authors felt that it was important to keep the communications cable intact to remove the possibility of it being disconnected from the riser system at the T-connector.

Test lab: mechanical arrangements, signal testing (distance tests and quality), mounting procedure, calibration procedures, environmental testing (pressure).

\section{B. Riser Components}

The large scale model riser is made from a number of important components and these are outlined in the ensuing sections.

1) Riser: The flexible riser, made of durable and lightweight uniform polyethylene plumbing pipe, had the primary dimensions shown in Table I. These characteristics meant that the pipe was fairly lightweight and allowed for relatively easy manipulation and placement of the pipe before and after testing. The inner diameter of the pipe was large enough to ensure that instrumentation (discussed in section IIB.2) could be designed at low-cost, and the outer diameter was 


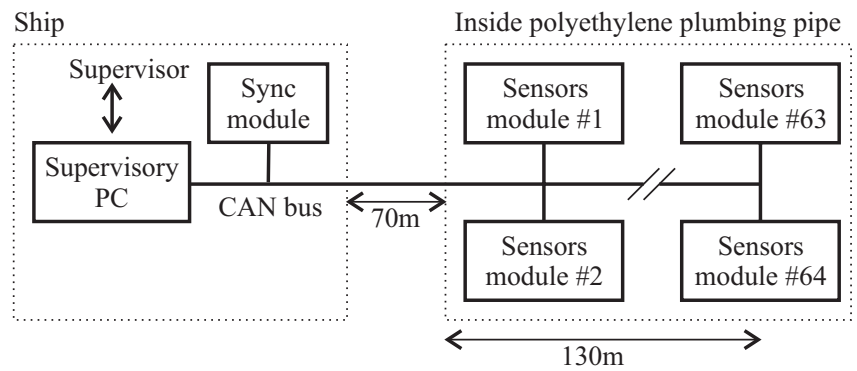

Fig. 1. Block diagram

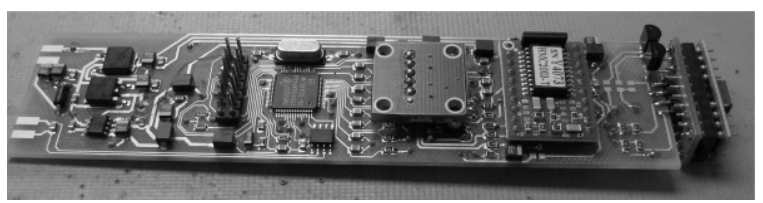

Fig. 2. Fully instrumented sensor module (acceleration, yaw gyro rate, and magnetic compass).

small enough to provide for large aspect ratio (length/diameter) experiments. Furthermore, the pipe had smooth inside walls, which provided little resistance when pulling the instrumentation through. Finally, the pipe was continuous, weather resistant, and water-tight from one end to the other, which provided additional protection to the instrumentation.

2) Instrumentation: A conceptual block diagram of the instrumentation system is shown in Fig. 1, where three main components can be identified: DSP-based sensor modules, synchronization module, and a supervisory PC (standard personal computer). A picture of the electronic module is shown in Fig. 2. Each module was equipped with a three-axis accelerometer, to determine acceleration data in the in-line and cross-flow directions. The slim profile of the accelerometers $(4 \mathrm{~mm} \times 4 \mathrm{~mm}$ $\mathrm{x} 1.45 \mathrm{~mm}$ ) made them attractive from the point of view of trying to fit the entire system inside a small diameter pipe. The electronic modules were also equipped with a sensor for yaw gyro rate, and a magnetic compass.

The selected hardware architecture provides the flexibility required to perform real-time digital signal processing within each module. The connection between the modules and the computer is performed through a Controller Area Network (CAN) bus that ensures robust high-speed data transmission even under noisy electrical environments. The system is synchronized by a sync module to ensure accurate simultaneous data acquisition as indicated in Fig. 1. After each conversion, the data packets containing motion information are transmitted in real-time through the communication bus. The software used for this research was developed in-house to monitor and collect the data coming from the modules, and display the evolution of the riser with a user interface. It enabled the user to set the sampling frequency, the duration of sampling in seconds, and also gave the user the ability to manually start and stop an experiment.

As can be deduced from the block diagram in Fig. 1, the concept of modularity is one of the key features of the

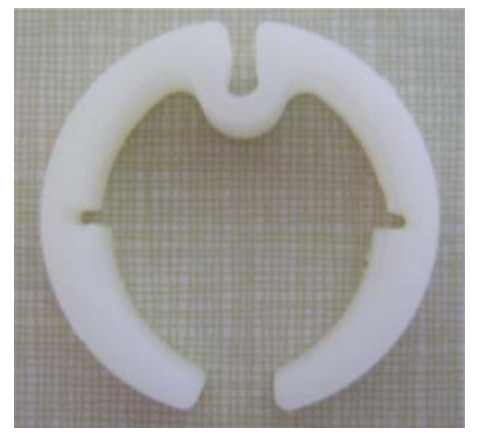

Fig. 3. Placement Puck

proposed measurement system. The modular architecture of the system allows increasing the number of modules to achieve higher mapping modes with the additional advantage of low cost per module. A detailed explanation of this advanced measurement system can be found in [7].

3) Placement Pucks: Plastic placement pucks, shown in Fig. 3, were designed for the model riser to keep the modules near the center of the pipe. The outer diameter was made to be slightly smaller than the inner diameter of the pipe and the inner diameter of the puck was large enough to fit the power and communication cables under the module when mounted. The bottom of the puck was cut out so that additional pucks could be added anywhere along the length of the cables.

Also, the puck had a small key hole at the top where a coated wire rope would be attached to hold the module in place along the length of the pipe.

4) Coated Wire Rope: The coated wire rope is a $1 / 8$ common clothes line, rated for up to $1100 \mathrm{lbs}$ of tension. The wire rope was snapped into the key hole of the placement pucks, and clamped at each end. This produced a way for the modules to be pulled through the pipe without having to pull on any of the cables.

5) Power and Communication Cables: Two sets of cables were used for the LS Model Riser. The first set, or the internal set, was used for connecting modules to one another inside the model riser, and the second set, or the external set, was spliced into the internal set to connect the riser to the data acquisition system onboard the vessel. The internal set consisted of a category 5 network cable and a group of 4 (10 AWG) submersible power cables. The external set consisted of a category 5 network cable and a 10-4 CabTire (10 AWG, 4 Conductor) power cable.

\section{Assembly}

With the components selected, the test laboratory was used to establish assembly procedures for the model riser system. Fig. 4 shows the assembly of a single sensor module. The wire rope is tensioned over the length of the riser and hose clamps are used to securely fasten the power and cat5 cables to it. The cat5 cable cannot be seen in Fig. 4 because it was decided to place it in the middle of the power lines to ensure that the pressure from the hose clamps did not compromise the network cable. Placement pucks hold the sensor module 


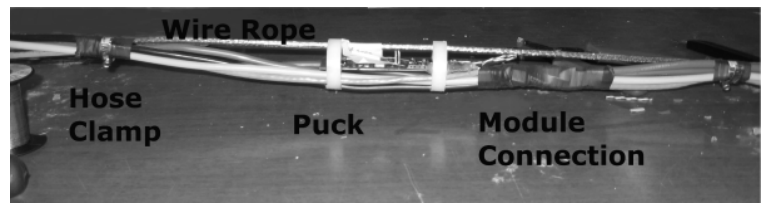

Fig. 4. Placement Puck

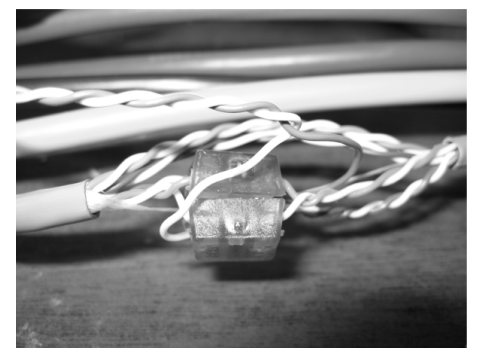

Fig. 5. Cat5 Tap Connector

in place and provide a channel for the power and network cables to fit underneath it. Power is supplied to the module by soldering one of the power cables to it, and the communication cable is connected to the module via tap connectors. These connections are then tightly wrapped in vulcanizing tape to provide another layer of protection and to prevent them from getting caught or hooked inside the pipe. Figure 5 shows the tap connector joining twisted pairs from the sensor module to a corresponding pair in the cat5 cable. Each component connected to the riser system was mounted in a similar manner according to written guidelines for the exact placement of modules, pucks, and clamps. For an experiment of this scale this made troubleshooting easier, since the layout of each board was identical at different location. In addition to this, a color-coded template was developed to dictate to which of the two power cables, and four cat5 twisted pairs each module must be connected. This was necessary to ensure that the modules were correctly staggered along the length of the riser, to provide the redundancy described earlier.

In order to assemble the riser two items were necessary: a building large enough to lay the riser straight, and a suitable work space that could also extend over the length of the riser. The National Research Council Canada's Institute for Ocean Technology (NRC-IOT) donated a long underground corridor approximately $200 \mathrm{~m}$ in length for the storage and assembly of the model riser. It was necessary to lay the instrumentation out straight, rather than coiling to avoid entanglement and to ensure that the orientation of the power bundles, tension rope, and sensor modules was similar throughout the length of the riser. This made calibration easier, and also made it possible to visually inspect all of the modules in the string at the same time. Furthermore, the arrangement also made it possible to pull the instrumentation through the riser with minimal resistance.

The sensor mudules were connected using a mobile work station, as shown in Fig. 6. This consisted of a working surface with a set of clamps attached to either end. The

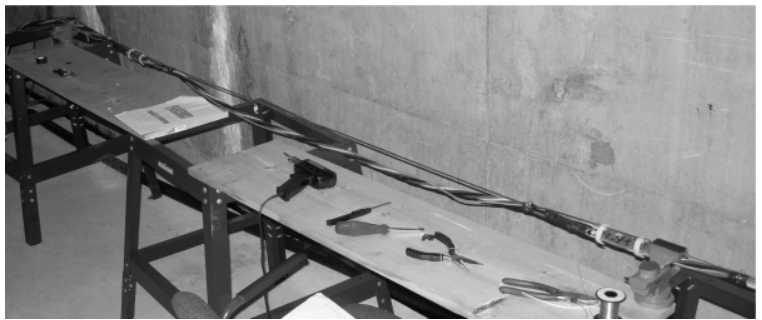

Fig. 6. Moving Assembly Table

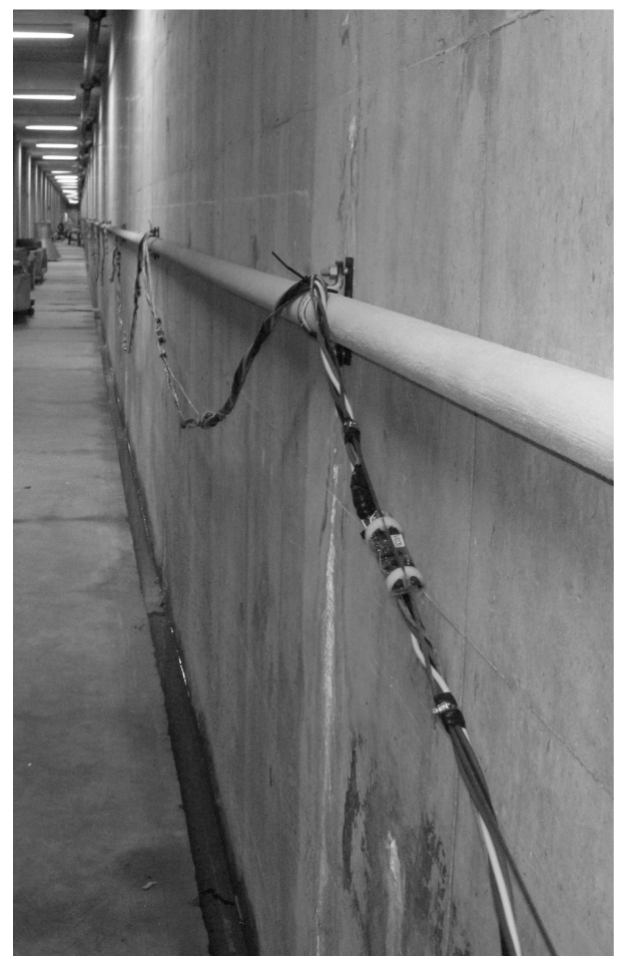

Fig. 7. Riser Modules Hanging in Corridor

wire rope was tensioned between the clamps and the power and network cables passed through a channel underneath the clamps. The module was positioned and mounted according to the procedures defined by the laboratory experiments. When mounting was complete, the entire work station was displaced by $2 \mathrm{~m}$ and the process was repeated for all of the remaining modules. As modules were mounted they were suspended above the floor (as shown in Fig. 7) to keep them protected, and prevent entanglement. Figure 8 shows the work station, and module string. Also shown is the pipe laid out behind the assembly table.

When all of the modules were mounted to the wire rope, the entire system was checked for consistency with the in-house data acquisition software, and the entire instrumentation string was pulled through the model riser. A conceptual illustration of a section of the instrumented riser is shown in Fig. 9.

\section{CALIBRATION}

In order to analyze the data delivered by the model riser, each of the sensor modules had to be calibrated. It would 


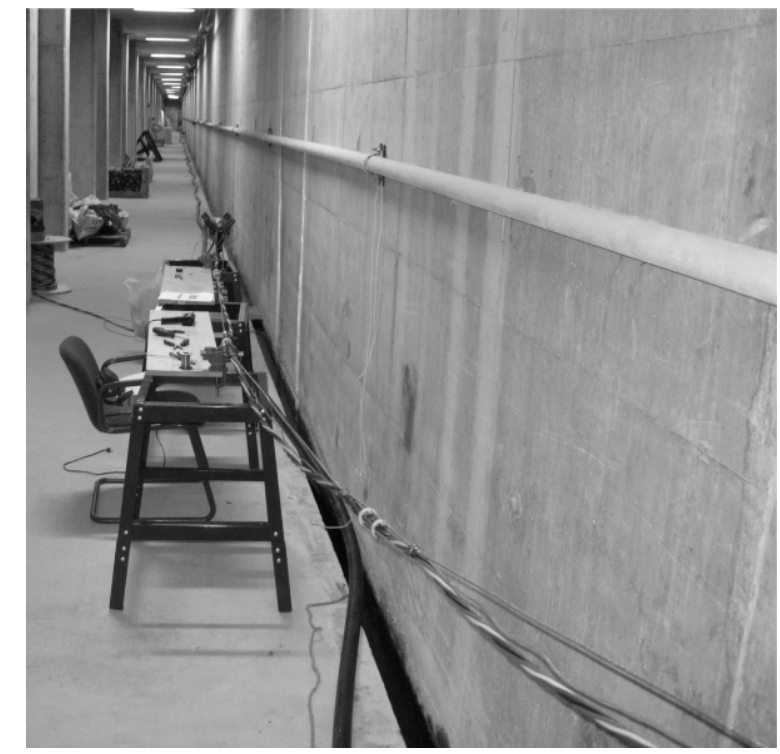

Fig. 8. Assembly Table and String of Modules

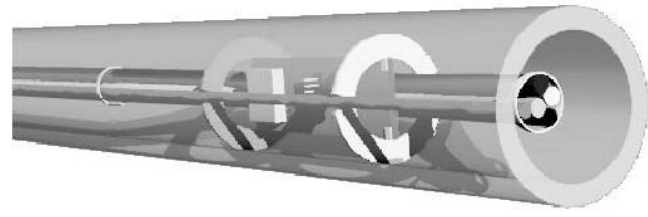

Fig. 9. Illustration of Instrumented Riser

have been very difficult, and costly, however, to construct a calibration rig capable of accurately calibrating the entire riser at once. So, it was decided to calibrate the riser in sections. Accelerometers are linear devices that can be calibrated by aligning their axis of sensitivity with and against the direction of gravity. Calibration is performed by reading the output from an accelerometer at equally spaced angular positions around a horizontal axis, where the axis of sensitivity is in a vertical plane.

Figure 10 shows the calibration rig designed for this research. The instrument is laid horizontal, and the pipe fixed into place using U-bolt type clamps, which have been scored at thirty degree increments from 0-360 degrees. The purpose of the calibration is to determine the sensitivity and offset of each accelerometer according to:

$$
A=s g \sin \theta+b,
$$

where $s$ is the sensitivity, $g$ is the gravitational acceleration, $b$ is the offset, and $\theta$ is the angle between the axis of the accelerometer and gravity (the local vertical). The output, $A$ is expressed in terms of $g$ or $\mathrm{m} / \mathrm{s}^{2}$ of acceleration

The entire model riser was calibrated piece-wise in this manner until the sensitivity and offset of each accelerometers was obtained.

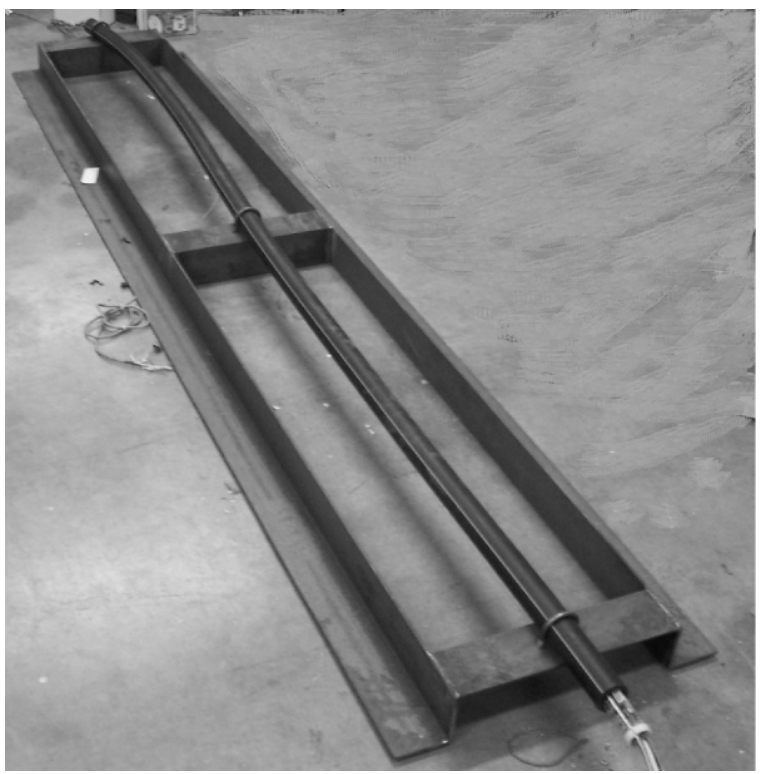

Fig. 10. Calibration Rig

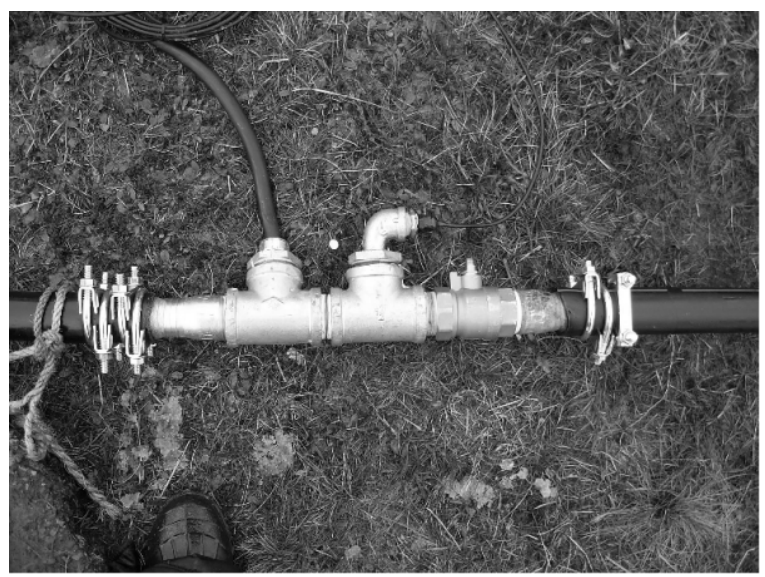

Fig. 11. T-Section

\section{External Mounting Components}

This system was designed to collect and display realtime data, so it is necessary to connect the power, gound, and communication cables from the inside of the riser to a computer and power supply located onboard the ship. This was accomplished by inserting a 'T-section' at the top of the riser through which the necessary cables could be passed.

Firstly, the 4 power cables had to be spliced into the 10-4 CabTire cables. The CabTire cable and cat5 network cable were then passed through the T-section and out through a watertight plumbing connection. Figure 11 shows the TSection fully assembled sitting in the pipe.

To map the frequency and amplitude response of the system the model riser will be suspended from a ship and towed at a number of velocities. External mounting hardware was therefore required. Wire mesh grips, shown in Fig. 12 were chosen as the method to enable the model riser to be suspended from a hook or other device. The grips work by tightening 


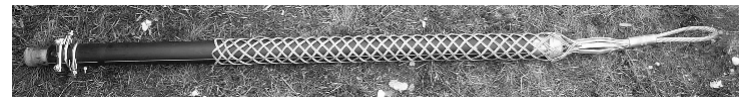

Fig. 12. Mesh Grip

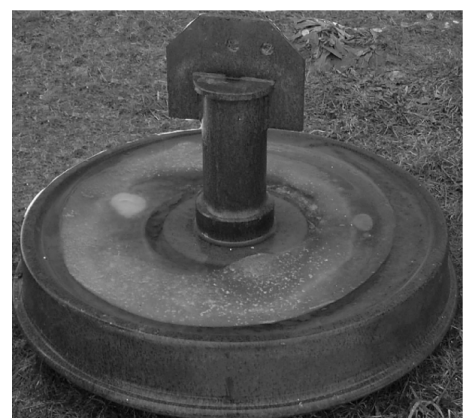

Fig. 13. Railway Wheel

as tension is applied at the end. However, in industry they are mainly used to pull wires and cables, which are able to take the force of the grips tightening, whereas the model riser pipe is hollow and therefore may crush under this pressure. To remedy this, solid PVC rods were made to fit inside the pipe at both ends. This gave the pipe the strength to withstand the force from the grips.

In order to weigh down the system and ensure the riser remained as close to vertical as possible, it was determined that a railway wheel should be attached to the bottom section of the model riser. The wheel, shown in Fig. 13 weighs approximately $363 \mathrm{~kg}$ and has a volume of approximately 0.05 $\mathrm{m}^{3}$. In addition, a swivel wheel was necessary to prevent the riser from spinning in the event that the wheel spins in the water as the model is being towed. The swivel was attached to a separation plate, shown in Fig. 14 with connections for the riser, wheel, and a winch line from the vessel.

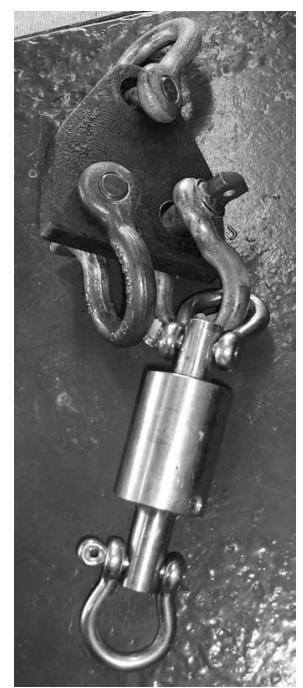

Fig. 14. Separation place, shackles and Swivel

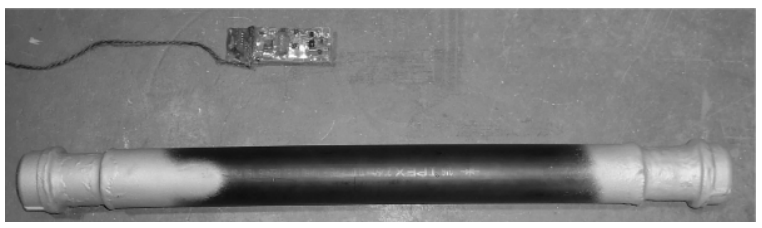

Fig. 15. Samples for Pressure Tests

\section{A. Environmental Testing}

Due to the nature of these tests, catastrophic failure of the aparatus could result in loss of the entire system to the ocean. Each of the riser components, therefore, needed to be tested under realistic operating conditions. The following tests were performed (in addition to the battery of laboratory tests) to mitigate against such undesirable consequences.

1) Tension Tests: Preliminary numerical analysis performed by [6] indicated that the maximum tension that the pipe would experience would be $10 \mathrm{kN}$. A tension of 14,000 N was applied to the pipe which established that the strength of the pipe was more than enough to withstand testing.

2) Pressure Tests: The components of the model riser were expected to operate at a maximum water depth of $130 \mathrm{~m}$. Therefore, two tests were performed in a pressure chamber. The first test consisted of placing an electronic sensor in the pressure chamber and testing at 290 psi (equal to roughly 190 $\mathrm{m}$ water depth) for 10 minutes. The sample was then tested using the data acquisition system and results showed that it continued to function correctly.

A similar test was performed on the empty pipe, and results showed that it did not plastically deform at the given pressure. Subsequent testing showed that the pipe experienced visible plastic deformation at $350 \mathrm{psi}$, which corresponded to a depth of roughly $230 \mathrm{~m}$ (much deeper than the $130 \mathrm{~m}$ water depth intended for these tests). Figure 15 shows the test samples that were used in the pressure tests.

3) Testing the Mesh Grips: To ensure that the grips worked properly, that they would not slip off of the pipe, and that the plugs were sufficiently strong, a test was performed in the Strength Lab in the Engineering Building at Memorial University. The set up can be seen in the figure below. The plugs were placed inside a sample of the Gold Stripe IPEX pipe which was capped with common steel plumbing caps and then the grips were placed over both ends. One end was then bolted to the floor while the other end was attached to an overhead crane, equipped with a load cell, and lifted until the desired tension was reached. As shown in previous calculations, the expected tension the riser will experience is approximately $10,000 \mathrm{~N}$. Therefore, to err on the side of caution, approximately $14,000 \mathrm{~N}$ was applied to the set up and not only did the grips hold, they did not move or slip on the pipe, the pipe did not crush under the intense force of the grips and the caps did not move and went undamaged. Therefore, it was felt that the grips, plugs and caps were suitable for use in this project. 


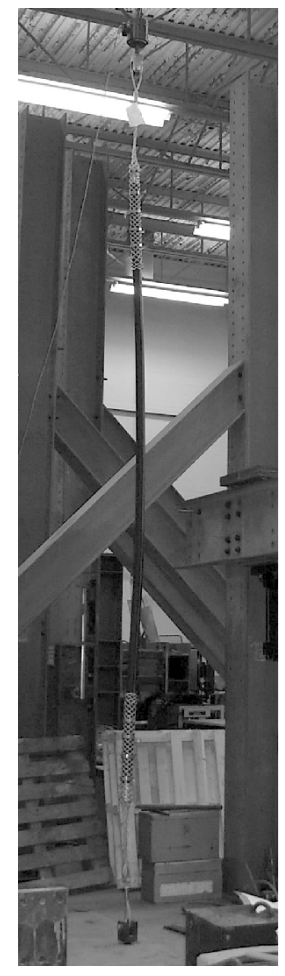

Fig. 16. Mesh Grip Strength Test Setup

\section{B. Proposed Field Tests}

Field testing for the model riser is scheduled for Fall 2007 off the coast of St. Johns, Newfoundland in a natural trench called The Cordelia Deeps. The pipe will be towed at speeds ranging from 0.15 to $1.50 \mathrm{~m} / \mathrm{s}$. Two different types of test will be done:

1) steady state runs where 3 minutes of data will be recorded at $15 \mathrm{~Hz}$ after a steady state speed has been reached. At each speed, two 3 minute samples will be taken to give some repeatability to the tests.

2) transient runs where the vessel is either speeding up or slowing down.

Table II lists the run number and current speed for steadystate test to be carried out. The transient test cases will consist of a steady acceleration from a velocity of 0.15 to $1.5 \mathrm{~m} / \mathrm{s}$, followed by a steady deceleration back to $0.15 \mathrm{~m} / \mathrm{s}$. The results of these tests will be entered into in-house data analysis codes and analyzed for multi-modal vibrations and displacement amplitudes.

\section{CONCLUSION}

For this research a densely instrumented large scale model riser was designed to gather vortex-induced vibration data at high mode number.

The design philosophy for the model riser was built on reliability, robustness, and redundancy. These tenets led to the fabrication of a relatively inexpensive model with heavily protected instrumentation, that operated over four distinct communication channels and two power cables.
TABLE II

Steady State Test Plan

\begin{tabular}{|c||c|}
\hline Run No. & Velocity \\
\hline 1 & 0.15 \\
\hline 2 & 0.45 \\
\hline 3 & 0.75 \\
\hline 4 & 1.25 \\
\hline 5 & 0.9 \\
\hline 6 & 0.6 \\
\hline 7 & 0.3 \\
\hline 8 & 1.35 \\
\hline 9 & 1.45 \\
\hline 10 & 1.50 \\
\hline 11 & 1.45 \\
\hline 12 & 1.40 \\
\hline 13 & 1.30 \\
\hline
\end{tabular}

Furthermore, an innovative measurement system for realtime characterization of VIV was developed. This system was modular and allowed for additional sensor modules to be added without sacrificing the integrity of the data.

A fully functional large scale riser model was developed, that will serve as a test platform for other areas of VIV research, such as fatigue and buckling analysis, various realistic catenary shapes and vibration suppression devices.

\section{ACKNOWLEDGMENT}

The authors would like to thank the Natural Sciences and Engineering Research Council (NSERC) of Canada and Petroleum Research Atlantic Canada (PRAC) for their financial contributions. Furthermore, the in-kind contributions of the National Research Council Canada-Institute for Ocean Technology, Oceanic Consulting Corporation, and Chevron Energy Technology Company are greatly appreciated. Finally, the design advice supplied by Charles Humphrey is gratefully acknowledged.

\section{REFERENCES}

[1] J. Vandiver, C. Mazel "A field study of vortex-excited vibrations of marine cables," Proceedings of the 1976 Offshore Technology Conference, Paper NO. 2491, Dallas, 1976.

[2] J. Vandiver, "Drag Coefficients of long flexible cylinders," Proceedings of the 1983 Offshore Technology Conference, PaperNO. 4490 Houston, 1983.

[3] Y. Kim, J. Vandiver, "Vortex-Induced Vibration and Drag Coefficients of Long Cables Subjected to Sheared Flow," Proceedings of the 4th OMAE Symposium, Volume One, ASME, Dallas, Texas, February 1985

[4] J. Vandiver, T. Chung, "Predicted and measured response of flexible cylinders in sgeared flow," Proceedings of ASME Annual Meeting Symposium on Flow-Induced Vibration, Chicago, 1988. [Kim et al. (1983)]

[5] J. Vandiver, H. Marcollo, S. Swithenbank, V. Jhingran, "High mode number vortex-induced vibration field experiments," Proceedings of the 2005 Offshore Technology Conference, Paper No. 17383, Houston, 2005.

[6] G. Stone, (private communication), 2007.

[7] M. Ordonez, M.O. Sonnaillon, D. Murrin, N. Bose, and W. Qiu, "An Advanced Measurement System for Vortex-Induced-Vibrations Characterization in Large-Scale Risers," Proceedings of the 2007 IEEE OCEANS, Vancouver, 2007. 